\title{
Demographic and clinical characteristics of BPPV patients: a retrospective large cohort study of 1599 patients
}

\begin{abstract}
Benign Paroxysmal Positional Vertigo (BPPV) is one of the most common peripheral vestibular problems, characterized by intense positionally provoked vertigo with a brief duration. However, studies of BPPV with demographic analyses are scant. To our knowledge, this is the largest retrospective analysis of BPPV patients seen in a 5-year period (2015-2019). The aim of this study is to document the demographic data of BPPV patients and describe the distribution of age, gender, the most common form and affected ears, associated comorbidities, type of treatment used, treatment efficacy, recurrence rates, prevalence of falls and postural instability. Predisposition for right posterior canal BPPV is almost unequivocally consistent in the literature. Hypertension is significantly associated with the occurrence of BPPV. The lower incidence of horizontal canal in this study may be explained by higher rates of spontaneous resolution and the exclusion of iatrogenic horizontal BPPV due to migration, while the lack of anterior canal BPPV was due to exclusion of atypical variants. There is also a high prevalence of falls and postural instability in BPPV patients, which requires further study. The demographic analyses are consistent with the literature, however treatment efficacy based on type of maneuver used, the influence of BPPV and its treatment on postural stability have not been sufficiently addressed. In this study, there is a high prevalence of self-reported falls $(47.7 \%)$ and more than half of the patients $(54 \%)$ with abnormal postural stability. Future prospective studies are necessary to validate such findings.
\end{abstract}

Keywords: BPPV, demographic, characteristics, cohort, falls, postural, stability, recurrence, treatment, efficacy
Volume 12 Issue I - 2020

\author{
Chua KWD, ${ }^{1,2}$ Gans RE,' Spinks S' \\ 'The American Institute of Balance (AIB), United States \\ ${ }^{2}$ Depart of Otolaryngology, Changi General Hospital (CGH), \\ Singapore
}

Correspondence: Kenneth Chua, Department of Otolaryngology, Allied Health (Audiology), Changi General Hospital EHN 2B, 2 Simei St 3,Tel +656936526I, Singapore, Email Kennath_chua@cgh.com.sg

Received: December 17, 2019 | Published: January 21, 2019
Abbreviation: GRM, gans repositioning maneuver; IQR, inter-quartile range; SLM, semont liberatory maneuver; RPC-HC, right posterior canal-horizontal canal; MCRM, modified canalith repositioning maneuver; $\mathrm{CRP}$, canalith repositioning maneuver; HHM, horizontal hybrid maneuver; LPC-HC, left posterior canalhorizontal canal; PC-BPPV, posterior canal-benign paroxysmal positional vertigo; AC-BPPV, anterior canal- benign paroxysmal positional vertigo; CDP, computerized dynamic posturography GANS-SOP, GANS sensory organization and performance; ADLs, activities of daily living; HRQoL, health-related quality of life

\section{Introduction}

Benign Paroxysmal Positional Vertigo (BPPV) is the most common form of vertigo ${ }^{1,2}$ and happens when otoconia is displaced into one, or more semi-circular canals. Dislodged otoconia may adhere to the cupula (cupulolithiasis) ${ }^{3}$ or more commonly be free floating (canalithiasis) in the canal as evidenced by intraoperative findings $\mathrm{s}^{4}$. BPPV is characterized by an intense brief duration of vertigo when the patient's head is moved into a gravity dependent position and most commonly affects the posterior semicircular canal due to its physical location in respect to the utricle. ${ }^{5}$ Spontaneous resolution of BPPV is highly variable ranging from $20-80 \%$ at one month after diagnosis ${ }^{6}$ but is difficult to predict due to the heterogenous etiology and controversial pathophysiology of this condition. ${ }^{7}$ Although benign in nature, BPPV can negatively impact health-related quality of life (HRQoL), causing physical and psychological problems and impacting activities of daily living (ADLs). ${ }^{8} 9$ Patients with BPPV may also be at elevated fall risk with actual falls leading to further disability and morbidity. In the senior age group, the fear of falling may also immobilize them resulting in secondary comorbidities. Thus, accurate diagnosis and prompt treatment of BPPV is warranted to reduce complications such as fractures and muscle-wasting from falls and immobility. ${ }^{10}$

While medication is ineffective for BPPV, treatment maneuvers on the other hand have been proven to be highly efficacious with most patients in complete remission after 2 treatments. ${ }^{11-14}$ However, clinically, patients with BPPV present with different medical history and comorbidities, which may influence the etiology, type of maneuvers used, recurrence rates, outcomes of treatment, number of treatments required and associated symptoms. Hence, treating BPPV patients may not be a straight-forward process despite the well-described simple to perform maneuvers. Demographic analyses of BPPV hence, underscore the importance to better understand the profile of this group of patients in order to plan and make the most efficacious diagnosisbased rehabilitation strategies and recommendations to at risk population. To our knowledge, this is one of the largest retrospective analyses of BPPV patients seen in a five-year period (2015-2019). The aim of this study is to characterize BPPV through the retrospective analysis of age profile, gender distribution, most common form and type, associated comorbidities and clinical characteristics, which are all important factors for the accurate diagnosis and delivery of optimal treatment plans and follow-up recommendations for patients with BPPV. 


\section{Materials and methods}

\section{The study sample}

We retrospectively reviewed the charts of patients who were seen at the American Institute of Balance (AIB) from January 2015 to $31^{\text {st }}$ June 2019. The charts were filtered for the international classification of disorders-10 (ICD-10) diagnosis coding and we identified 1542 patients who were diagnosed with BPPV, confirmed by clinicians who have had extensive training in vestibular and equilibrium studies and with video-oculography recordings. 57 patients were negative for BPPV by the time they presented to the clinic. The patients' charts were reviewed to extract information such as age, gender, side of involvement, type of treatment maneuver used, number of treatment sessions required, outcomes, recurrence, postural study results, comorbidities from self-reported medical history (cross-checked with medication list to ensure completeness and accuracy), history of falls, head trauma/concussion and fear of falling from completed patient questionnaires.

\section{Diagnosis, inclusion and exclusion criteria}

All 1542 patients had undergone vestibular function testing, which included; videonystagmography (VNG) and kinetic-rotatory chair (KRC); step-velocity test (RVS). Most of the patients also had advance testing with cervical-vestibular evoked myogenic potential (c-VEMP), postural study with either the GANS Sensory Organization and Performance test (GANS SOP) or Sensory Organization Test (SOT), with some patients who completed the video-head impulse test (vHIT). All patients had findings that were negative for any acute/ uncompensated peripheral vestibular involvement as evidenced by the absence of pathological nystagmus in any of the sub-tests and symmetrical labyrinthine reactivity in the KRC, c-VEMP and/or vHIT. Patients with an ICD-10 diagnosis coding for labyrinthine dysfunction were not included in this study. Diagnosis of BPPV was based on a combination of patient's history, subjective symptoms and objective paroxysmal torsional up-beating nystagmus with the torsional component towards the undermost ear during modified Dix-Hallpike maneuver for Posterior-Canal BPPV (PC-BPPV). ${ }^{15}$ Horizontal-Canal BPPV (HC-BPPV) was identified by either a paroxysmal geotrophic (towards the undermost ear) or ageotrophic (away from the undermost ear) nystagmus in a lateral recumbent position. Down-beating and other atypical nystagmus were not included in this study as there could be several confounding reasons for this observation including central non-fatiguing nystagmus, ${ }^{16}$ positional nystagmus in healthy individuals, ${ }^{17}$ atypical variants of PC-BPPV,${ }^{18}$ central adaptation to $\mathrm{BPPV}^{19}$ and rarely Anterior Canal-BPPV (AC-BPPV). ${ }^{20}$ Hence, of the 1542 patients, none were diagnosed with AC-BPPV. If there were different patterns of nystagmus suggesting more than one type or side of canal involvement, they were categorized as mixed-type BPPV or bilateral PC-BPPV. All patients with a history or clinical findings suggestive of a Central Nervous System (CNS) involvement or with non-paroxysmal nystagmus were excluded from this study. Patients with symptoms and history of BPPV but with no objective recordable nystagmus (sub-clinical BPPV) on the first presentation were also excluded from this study.

\section{Treatment}

PC-BPPV were treated with either Modified Canalith Repositioning Maneuver (MCRM), ${ }^{12}$ Semont Liberatory Maneuver (SLM) ${ }^{13}$ or Gans Respositioning Maneuver (GRM) $)^{14}$ When a single-treatment maneuver didn't work, a combination of maneuvers was employed. All HCBPPV were treated with the Horizontal Hybrid Maneuver (HHM), ${ }^{21}$ which treated both sides and types of HC-BPPV without the need for clinicians to repeatedly provoke the patients in order to accurately identify the site of lesion. Mixed-type BPPC were treated with Canalith Repositioning Procedure (CRP) for posterior canal followed by HHM. Sub-clinical BPPV during post-treatment modified Hallpike or at follow-up sessions were treated again on the symptomatic side. Patients are treated up to three times in one session, according to AIB's protocol. No mastoid vibration or recommendation of medication was given throughout the treatment sessions. All patients had follow-up visits at a 5-7 days interval. Modified Hallpike was performed at every session, and treatments were carried out if patients were still positive for BPPV. The procedures of all repositioning maneuvers are well described in the literature. ${ }^{11-14}$

\section{Definition of recurrence}

Recurrence of BPPV was defined as a return of subjective and objective signs and symptoms of BPPV within 30 days, confirmed by modified Dix-Hallpike and video-oculography.

\section{Statistical analyses}

All data was analyzed with SPSS version 21.0. The analyses included non-parametric Mann-Whitney U test, Krustal-Wallis for non-parametric analysis of variance for continuous variables and chisquare test of association for dichotomous and categorical variables.

\section{Results}

\section{Demographic data for BPPV patients}

One thousand five hundred and forty-two patients $(\mathrm{n}=1542)$ were included in this study with age ranging from 18-98 years old. There were 1086 females (median age 70; Inter-Quartile Range (IQR) 18) and 456 males (median age 72; IQR 16; (Figure 1). Females were more affected than males in a 2.4:1 ratio and comparative nonparametric analysis of mean age between the two gender groups were not statistically significant ( $\mathrm{P}$ value $=0.05$ ). $90.4 \%$ of the BPPV patients $(1394 / 1542)$ were above the age of 50 , while $9.6 \%(148 / 1542)$ patients were below 50 years of age. It was very uncommon to have patients under the age of 20 years with BPPV; $0.2 \%$ (3/1542), while the most common age group for BPPV was between 71-80 years; $32.1 \%$ (495/1542); (Figure 2).

\section{Type of canal involved}

Posterior canal involvement was seen in 1,422 (92.2\%) patients of which, 1118 (78.6\%) were unilateral and $304(21.4 \%)$ were bilateral. Looking at the unilateral involvement alone, $672(60.1 \%)$ were rightsided ,446 (39.9\%) left-sided in a 1.5:1 ratio (60:40). $57(3.7 \%)$ patients had HC-BPPV, 63 (4.1\%) patients were seen with a mixedcanal type of BPPV and no patients in this study had AC-BPPV; (Figure 3). A right-sided preference was still seen even in the mixedcanal BPPV patients; 35 patients $(35 / 63 ; 55.5 \%)$ were right posterior and horizontal (RPC-HC) and $28(28 / 63 ; 44.4 \%)$ were left posterior and horizontal (LPC-HC).

\section{Prevalence of associated comorbidities (\%)}

The highest prevalence of self-reported comorbidity was hypertension $(803 / 1503 ; 53.4 \%)$, followed by migraine (423/1513; $28 \%$ ), head injury/concussion $(303 / 1242 ; 24.4 \%)$, cardiovascular 
disease $(319 / 1502 ; 21.2 \%)$, diabetes $(290 / 1502 ; 19.3 \%)$, anxiety $(289 / 1501 ; 19.3 \%)$, depression $(260 / 1501 ; 17.3 \%)$, stroke/TIA $(107 / 1501 ; 7.1 \%)$, heart attack $(60 / 1502 ; 4 \%)$ and hypotension $(53 / 1502 ; 3.5 \%)$; (Figure 4). Self-reported hypertension was significantly correlated with occurrence of BPPV in the age group 71-80 years. $\mathrm{P}$ value $=0.002$; Chi-square value $=9.84$, Phi coefficient 0.141 (small effect size), OR: 5.246 (95\% CI; 1.666, 16.513). Patients who had self-reported hypertension are 5.25 times more likely to also have BPPV compared to patients who did not report hypertension as a comorbid condition. All other reported comorbidities were not significantly associated with BPPV whether gender or agegroup stratification was applied. The association between reported comorbidities and occurrence of BPPV are summarized in the table below; (Table 1).

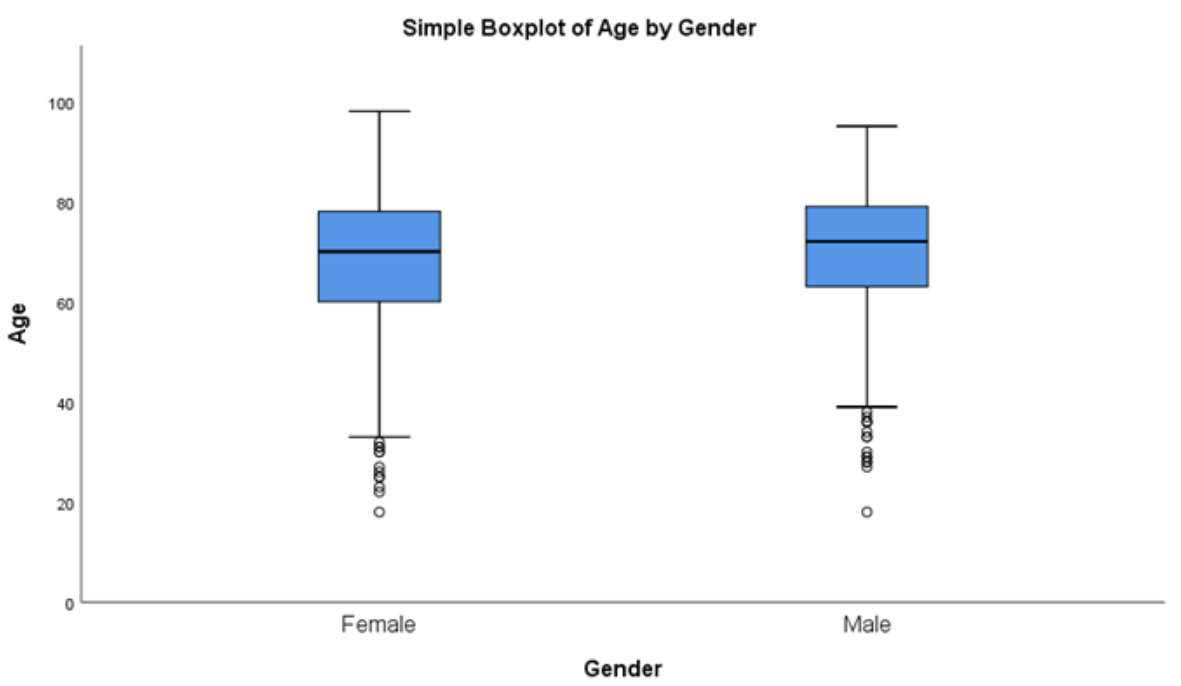

Figure I Box Plot of Gender distribution of BPPV patients.

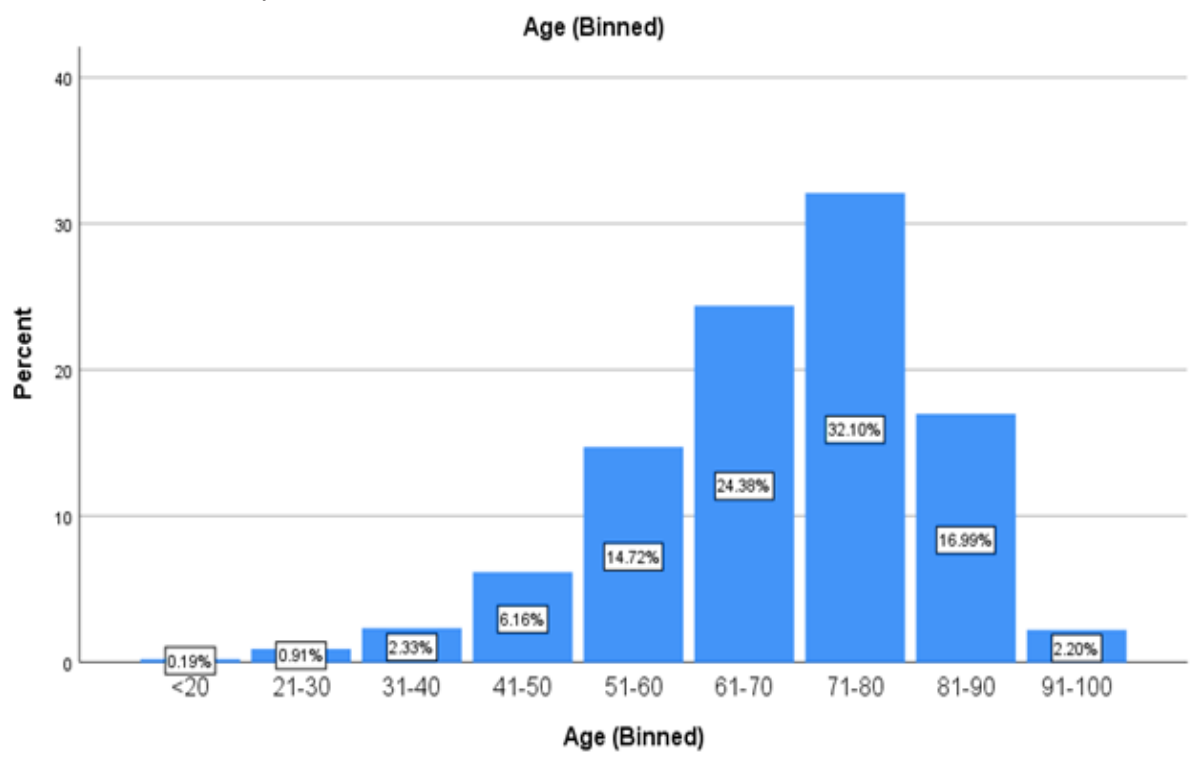

Figure 2 Histogram age distribution of BPPV patients.

\section{Demographic of treatment types, treatment sessions required and recurrence rate}

37 patients had missing information on type of treatment maneuver used. Of the remaining 1505 patients, 848 patients were treated with GRM (55\%), 194 with SLM (12.6\%), 99 (6.4\%), with MCRM, 191 (12.4\%), with a mix of 2 different maneuvers for PC-BPPV, 37 (2.4\%) patients with all three maneuvers for PC-BPPV performed, $56(3.6 \%)$ with HHM, 69 (4.5\%) with a mix of one maneuver for PC-BPPV and HHM, 7 patients $(0.5 \%)$ with a mix of 2 maneuvers and HHM and 4 patients $(0.3 \%)$ who required all three maneuvers for PC-BPPV with HHM (Figure 5). The median number of treatment sessions needed is one, with 1087 (72.2\%) patients requiring one treatment session for complete resolution. 288 (19.1\%) patients needed two sessions, 85 $(5.6 \%)$ patients needed three sessions and $45(3 \%)$ patients required 4 sessions or more. $91.4 \%$ of patients had resolution of BPPV after two 
treatment sessions and $97 \%$ of all patients were cleared within three treatment sessions. Recurrence rate was $16.9 \%(255 / 1505)$ in general and there was a significant association between number of treatment maneuvers used and recurrence of BPPV within 30 days; (Table 2). $(\mathrm{P}$ value $=0.000$; chi-square value $=66.83$; phi coefficient 0.211 ; OR:
3.2 (95\% CI 2.2-4.4). When more than 1 single-type of maneuver was required in the treatment session, there is 3.2 times greater odds of having recurrent BPPV, as compared to patients who had single-type treatment.

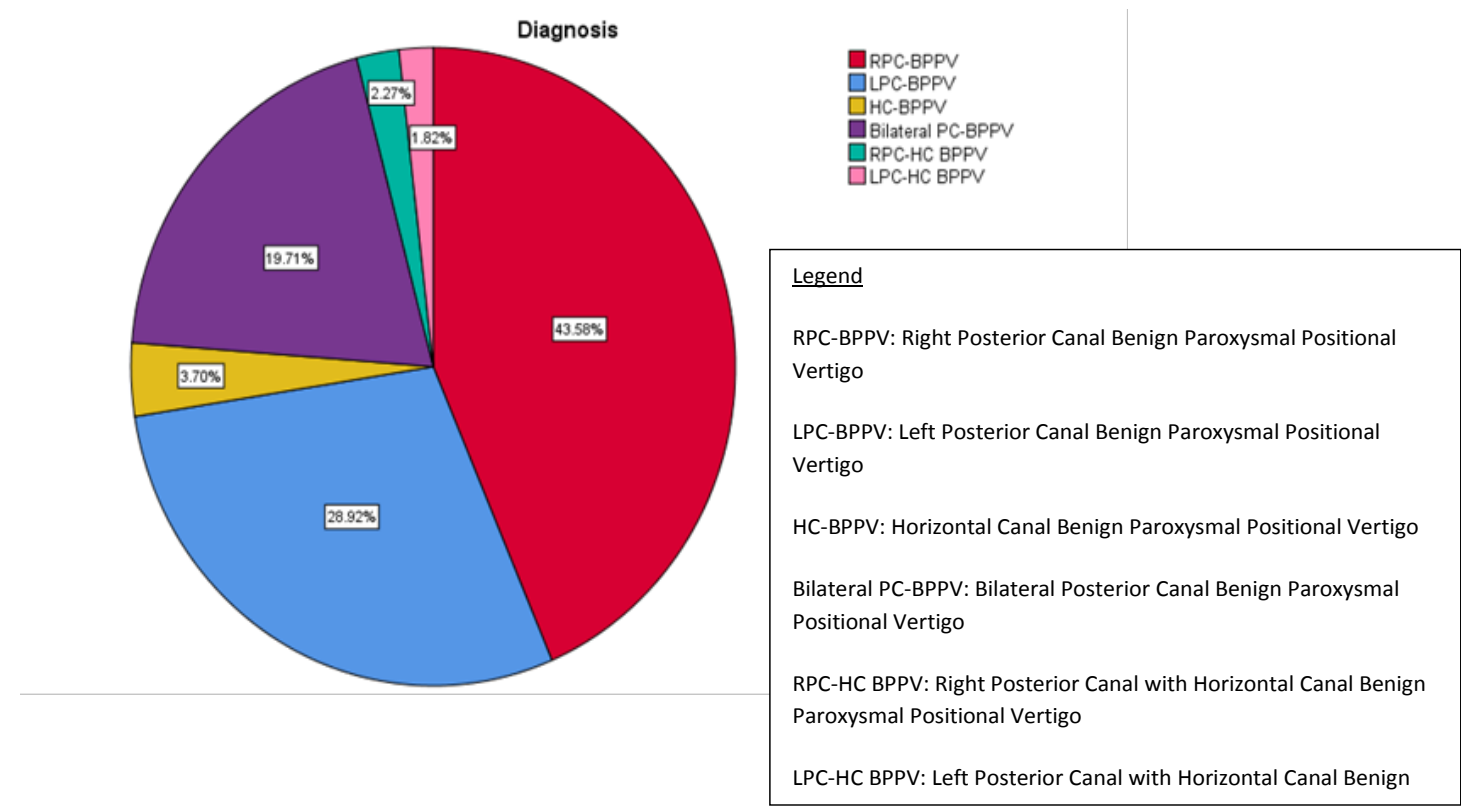

Figure 3 Type of canal involvement of BPPV patients.

\section{Associated Comorbidities}

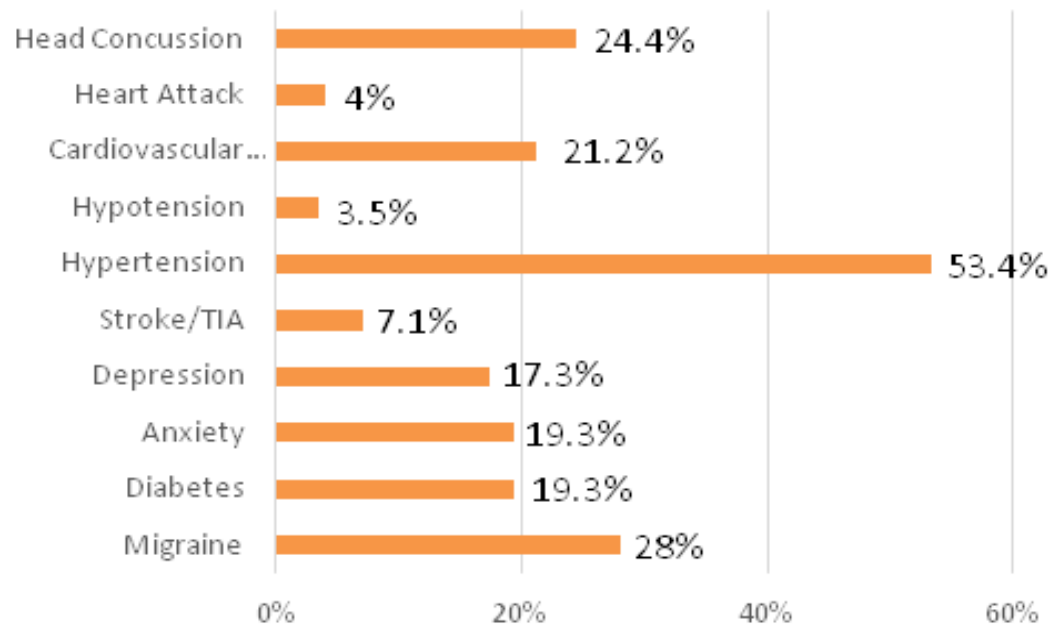

Figure 4 Prevalence of associated comorbidities.

\section{Prevalence of self-reported falls and abnormal postural stability in BPPV patients}

Of 1542 patients with BPPV, only 1447 responded to questioning of reported falls, 1193 responded to fear of falling and 1222 had available posturography results. $47.7 \%(690 / 1447)$ of patients reported having fallen at least once with $40.7 \%$ (486/1193) of patients who reported fear of falling. In 54\% (659/1222) of BPPV patients, abnormal posturography results were seen on either the Computerized Dynamic Posturography (CDP) or GANS Sensory Organization Performance test (GANS SOP). Of the 690 patients with reported falls, only $80.7 \%(557 / 690)$ reported the number of falls with a median of 2 falls (IQR; 2).; (Figure 6). There was no significant association between occurrence of BPPV with history of falls or fear of falling; (Table $3 \& 4$ ). Fear of falling was however significantly associated with self-reported falls with a moderate effect size. Patients with a fear of falling are 4.11 times more likely to have a reported history of falls as compared to those with no fear of falling reported; (Table 5). Occurrence of BPPV is not significantly associated with results of postural study; Table 6 or composite score of the Sensory Organization Test (SOT); (Figure 7). 
Table I Cross-tabulation of Associated Co-morbidities (Independent Variables) with BPPV occurrence (Dependent Variable), stratified by age-groups and gender

\section{Associated Co-morbidities (No/Yes)}

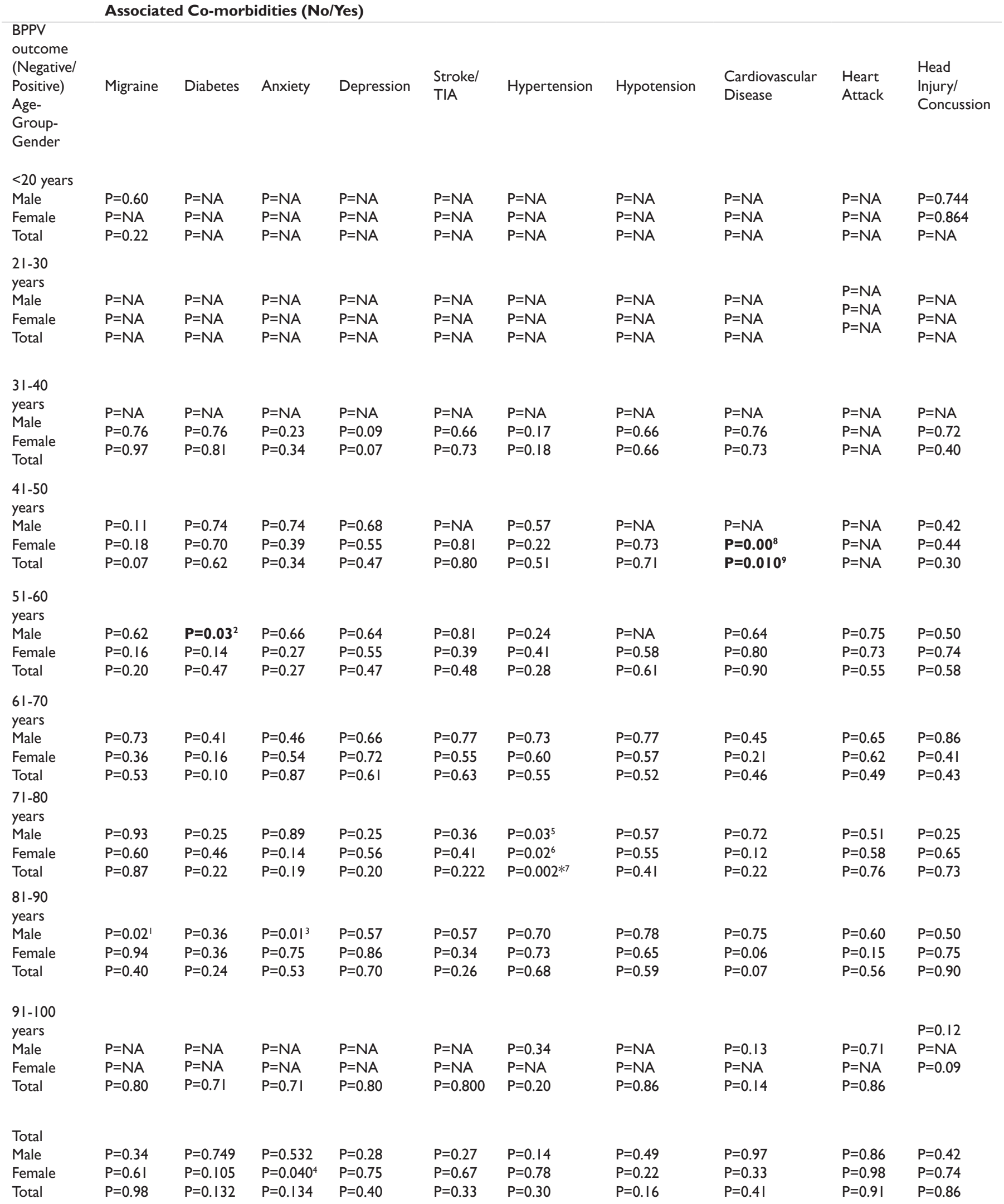

$150 \%$ of the cells have expected count less than 5 . Hence $p$-value is disregarded. 
$250 \%$ of the cells have expected count less than 5 . Hence $p$-value is disregarded.

$375 \%$ of the cells have expected count less than 5 . Hence p-value is disregarded.

${ }^{4}$ Chi-Square Value=4.2; phi coefficient 0.06 (very small effect size), OR: $3.2(95 \% \mathrm{Cl}$; I.0, I0.6). OR crosses I, hence disregard p value.

${ }^{5} 50 \%$ of the cells have expected count less than 5 . Hence $p$-value is disregarded.

$650 \%$ of the cells have expected count less than 5 . Hence p-value is disregarded.

${ }^{* 7}$ Chi-Square value=9.8; phi coefficient=0.14 (small effect size); OR $5.2(95 \% \mathrm{Cl}$; I.7, 16.5)

$875 \%$ of the cells have expected count less than 5 . Hence p-value is disregarded.

${ }^{9} 25 \%$ of the cells have expected count less than 5 . Hence p-value is disregarded.

Table 2 Cross-tabulation chi-square table of association between recurrence of BPPV and Number of Maneuvers used

\begin{tabular}{|c|c|c|c|}
\hline $\begin{array}{l}\text { Recurrence of Benign } \\
\text { Paroxysmal Positional } \\
\text { Vertigo within } 30 \text { days }\end{array}$ & $\begin{array}{l}\text { Number of Maneuv } \\
\text { I single maneuver }\end{array}$ & >I maneuver & Total \\
\hline NO & 1042 (83.4\%) & 208 (16.6\%) & 1250 \\
\hline YES & 155 (60.8\%) & $100(39.2 \%)$ & 255 \\
\hline Total & 1197 & 308 & 1505 \\
\hline
\end{tabular}

( $P$ value=0.000; chi-square value=66.83; phi coefficient $0.21 \mathrm{I} ; \mathrm{OR}: 3.2(95 \% \mathrm{Cl} 2.2-4.4)$

Table 3 Cross-tabulation chi-square table of association between occurrence of BPPV and history of falls

\begin{tabular}{llll}
\hline $\begin{array}{l}\text { Benign Paroxysmal Positional } \\
\text { Vertigo (NO/YES) }\end{array}$ & History of falls (NO/YES) & & YES \\
\hline NO & NO & $20(33.7 \%)$ & 53 \\
YES & $33(62.3 \%)$ & 690 & 1447 \\
Total & 757 & 710 & 1500 \\
\hline
\end{tabular}

$P$ value $=0.15$ (not significant)

Table 4 Cross-tabulation chi-square table of association between occurrence of BPPV and fear of falling

\begin{tabular}{llll}
\hline $\begin{array}{l}\text { Benign Paroxysmal Positional } \\
\text { Vertigo (NO/YES) }\end{array}$ & Fear of falling (NO/YES) & & Total \\
\hline NO & NO & YES & $19(45.2 \%)$ \\
YES & $23(54.8 \%)$ & $486(40.7 \%)$ & 42 \\
Total & $707(59.3 \%)$ & 505 & 1193 \\
\hline
\end{tabular}

$P$ value $=0.56$ (not significant)

Table 5 Cross-tabulation chi-square table of association between history of falls and fear of falling

\begin{tabular}{|c|c|c|c|}
\hline \multirow{2}{*}{ History of falls (NO/YES) } & \multicolumn{3}{|c|}{ Fear of falling (NO/YES) } \\
\hline & NO & YES & Total \\
\hline NO & $424(76.3 \%)$ & $132(23.7 \%)$ & 556 \\
\hline YES & 277 (43.9\%) & $354(56.1 \%)$ & 631 \\
\hline Total & 701 & 486 & 1187 \\
\hline
\end{tabular}

$P$ value $=0.00 ;$ Chi-Square $=128.0 \mathrm{I} ;$ phi coefficient $=0.33$ (moderate effect size); $\mathrm{OR}=4 . \mathrm{II}(95 \% \mathrm{Cl} ; 3.20-5.30)$

Citation: Chua KWD, Gans RE, Spinks S. Demographic and clinical characteristics of BPPV patients: a retrospective large cohort study of I599 patients.J Otolaryngol ENT Res. 2020; I2(I):20-30. DOI: 10.15406/joentr.2020.12.0045। 
Table 6 Cross-tabulation chi-square table of association between occurrence of BPPV and results of postural study

\begin{tabular}{|c|c|c|c|c|c|}
\hline \multirow{2}{*}{$\begin{array}{l}\text { Benign } \\
\text { Paroxysmal } \\
\text { Positional Vertigo } \\
\text { (NO/YES) }\end{array}$} & \multicolumn{5}{|c|}{ Results of Postural Study } \\
\hline & $\begin{array}{l}\text { Abnormal Sensory } \\
\text { Organisation Test }\end{array}$ & $\begin{array}{l}\text { Abnormal } \\
\text { GANS Sensory } \\
\text { Organisation Test }\end{array}$ & $\begin{array}{l}\text { NORMAL Sensory } \\
\text { Organisation Test }\end{array}$ & $\begin{array}{l}\text { NORMAL } \\
\text { GANS Sensory } \\
\text { Organisation Test }\end{array}$ & Total \\
\hline NO & $<5(6.5 \%)$ & 14 (30.4\%) & $5(10.9 \%)$ & $24(52.2 \%)$ & 46 \\
\hline YES & $222(I 8.2 \%)$ & $437(35.8 \%)$ & 127 (I0.4\%) & $436(35.7 \%)$ & 1222 \\
\hline Total & 225 & 451 & 132 & 460 & 1268 \\
\hline
\end{tabular}

$P$ value $=0.07$

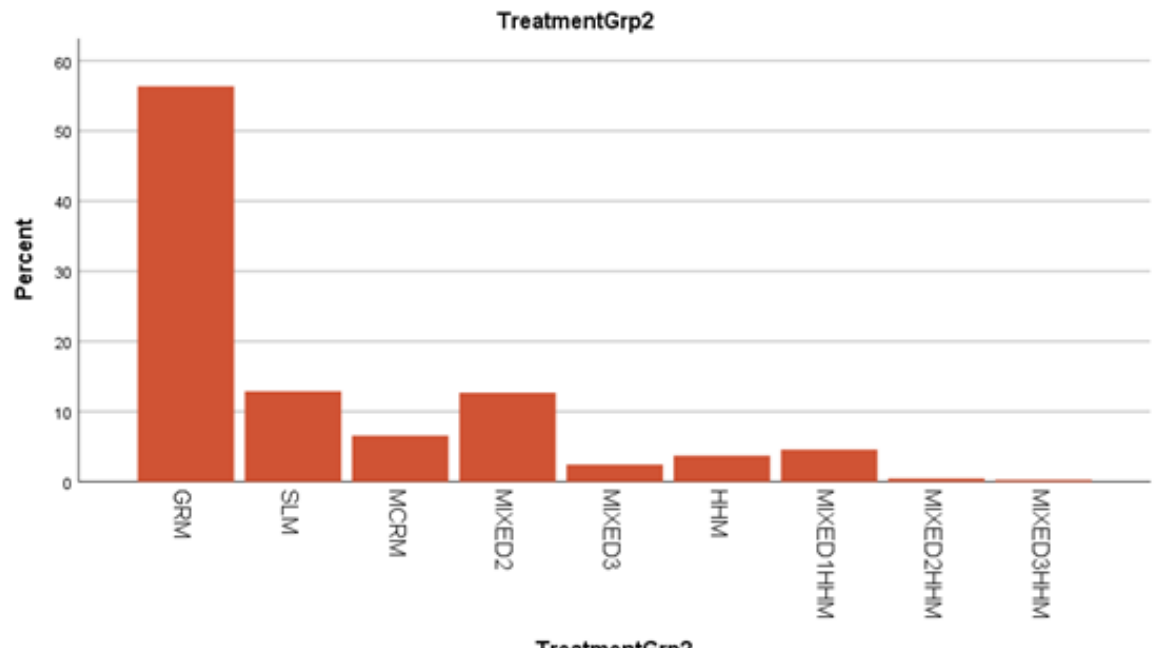

Figure 5 Distribution of treatment maneuvers used on BPPV patients.

$N=1505$

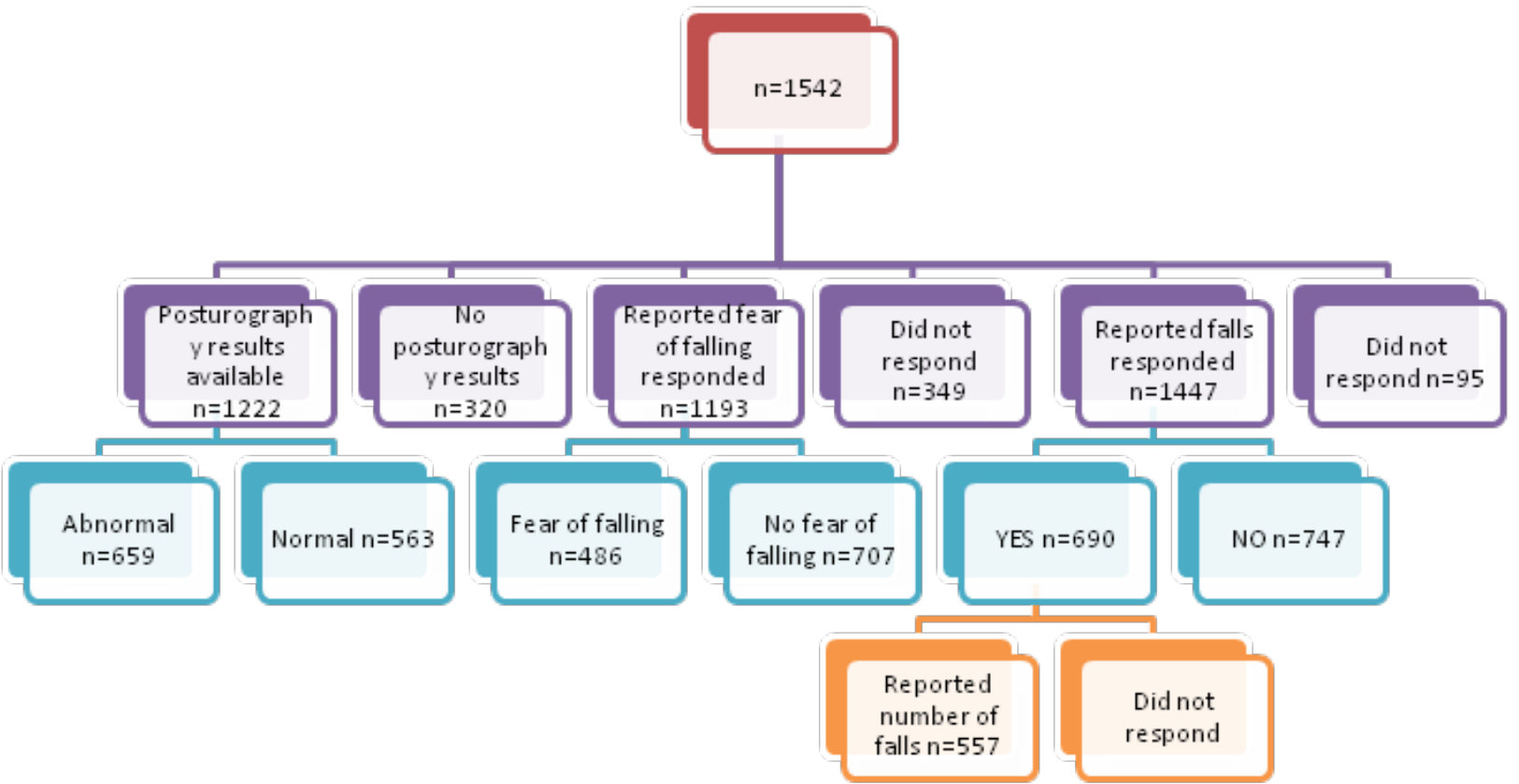

Figure 6 Demographic of self-reported falls, fear of falling and results of posturography in BPPV patients. 


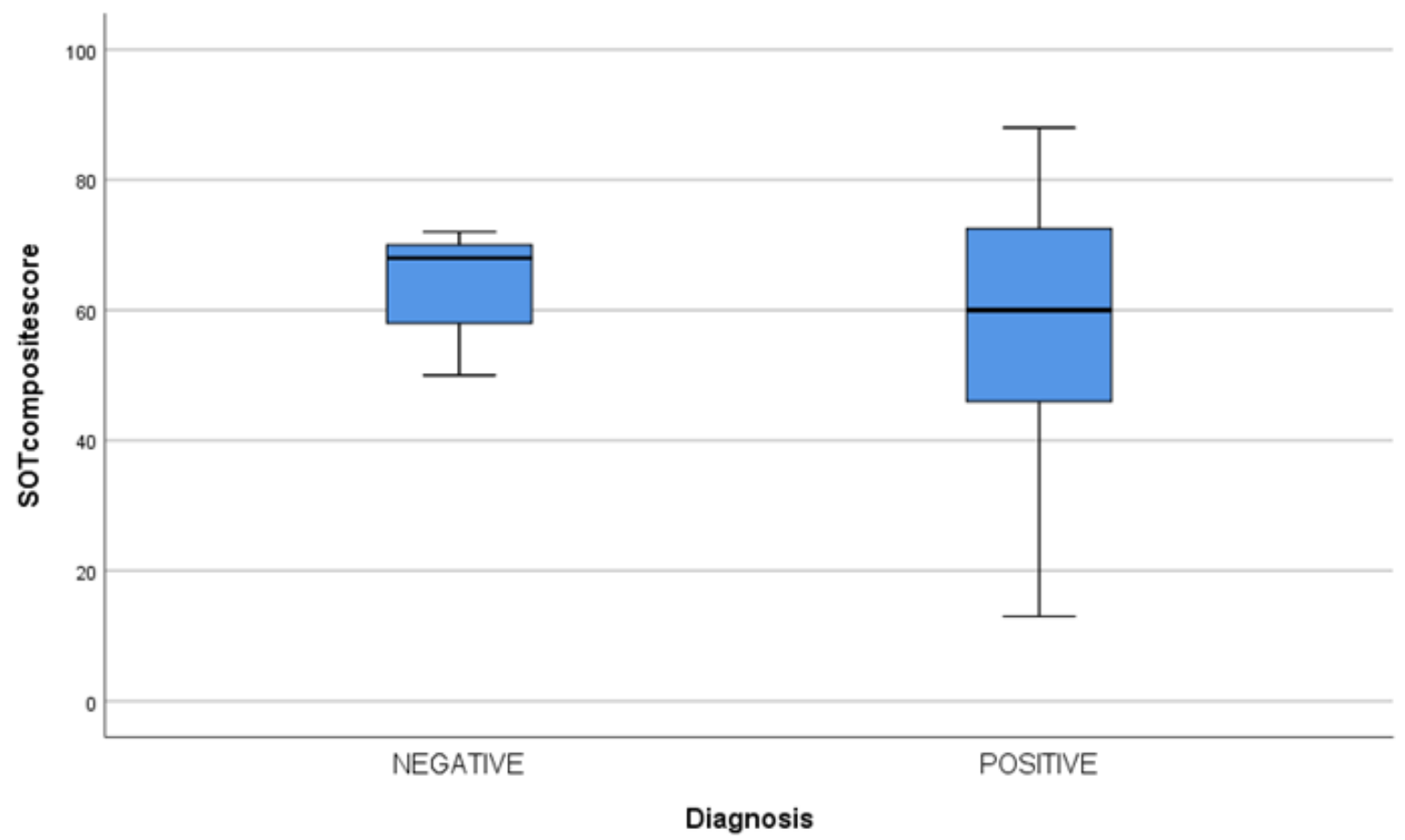

Figure 7 Mann-Whitney $U$ test of significance between SOT composite score and occurrence in patients with and without BPPV.

\section{Discussion and limitations}

Our results on the gender distribution of BPPV is congruent with other BPPV studies, which revealed a higher prevalence seen in females ranging from 1.9 to $2 .{ }^{22-24}$ Females in our studies are 2.4 times more likely to acquire BPPV than males. Most common type of BPPV is also of the posterior canal (92.2\%), affecting the right ear more than the left. This predisposition for greater occurrence of right posterior canal BPPV is almost unequivocally reported in the literature. ${ }^{25-30}$ Although it has been reported that the age of onset of BPPV is most seen between 50 to 70 years of age, ${ }^{30}$ our study revealed peaked age range to be from 71-80 years instead, which is consistent with reporting by Kao et al., ${ }^{22}$ but disagreeing with Yetiser et al's study with BPPV patients seen predominantly in the $3^{\text {rd }}$ to $5^{\text {th }}$ decade of life. ${ }^{31}$ The reasons for this discrepancy in age profiles may be partially explained by unrecognized BPPV in the elderly, who may often present with specific issues such as difficulty in obtaining an accurate history, restricted mobility and difficulty with diagnostic and treatment maneuvers due to vascular and orthopedic complications. ${ }^{32}$ It has been estimated by Oghalai et al. that unrecognized BPPV in the elderly is about 9 Of every 100 patients. ${ }^{33}$ However, with increasing recognition and improvement in diagnostic and treatment maneuvers, the age profile of BPPV patients may be shifting upwards due to greater recognition of BPPV in the elderly population. The age profile also depends on the geographical area and the type of patients the institute sees (pediatric/adult/veteran).

The AIB sees patients across the continuum of age and there was not a bias or a significant change in number of seniors age 65 and up according to Florida's population statistics in the period of this study's sampling. ${ }^{34}$ Hence, the demographic age profile of BPPV patients in this study is believed to be minimally biased and accurate. Although not well studied, the peak incidence of BPPV in this study may also be explained by differences in mobility with age. Seniors with BPPV and restricted/less mobility may have less spontaneous resolution as compared to younger mobile patients. BPPV rarely occurs in young adults less than 50 years of age (3.4\%), and it has been proposed that in addition to history of head trauma, ${ }^{29}$ younger individuals with comorbidities of migraine and Menieres Disease have higher incidence of BPPV. Unlike the study described by Moon et.al, we did not differentiate between geotrophic or ageotrophic forms of lateral canal BPPV. We also did not identify the side of involvement as all HC-BPPV patients were treated with HHM, which treats both sides and forms of HC-BPPV and does not require the clinician to identify the side of involvement. ${ }^{21}$ It has been anecdotally observed that patients with HC-BPPV are usually very symptomatic with strong autonomic responses, which includes nausea and possible vomiting. The authors in the study hence opined that it was impractical to have repeated provocation in order to identify the side and type of HCBPPV when outcomes of treatment with HHM does not depend on lateralization. Our proportion of HC-BPPV (3.5\%) was also different from other reports ranging from $1.3 \%^{35}$ to $11.9 \%{ }^{36}$ but was closest to Rukenstein's study. ${ }^{37}$ This could be explained by the stringent inclusion criteria for the diagnosis of HC-BPPV at only the first visit, excluding iatrogenic HC-BPPV from posterior canal migration by either the clinician after therapeutic treatment or the patients by selftreatment. Prevalence of HC-BPPV also depends on the interval from onset to diagnosis, which is negatively correlated. ${ }^{38}$ Consequently, studies with closer timeframe from onset to diagnosis usually report higher rates of BPPV. ${ }^{23}$ In our study, we did not record this time interval and hence, it is difficult to accurately compare the proportion of HC-BPPV with other studies. HC-BPPV is however, believed to be more self-limiting with high rates of spontaneous resolution and shorter symptom duration as compared to PC-BPPV. ${ }^{39-41}$ The natural position of the lateral canal facilitates spontaneous resolution when 
laterally turning in bed during sleep. Moreover, in our most common BPPV age group of 71-80 years, patients may be more sedentary and lying on the non-affected side for a longer period, effecting forceprolonged positioning. ${ }^{42}$

In this study, none of the patients had anterior canal BPPV, which is contradictory to other reports ranging from $1.9-21.2 \% .^{36-37}$ The diagnosis of AC-BPPV has always been challenging and requires good exclusion of central pathologies. ${ }^{23}$ Amongst other reasons, the higher occurrence observed in other studies may be atypical variants of BPPV as reported by Califano et al..$^{18}$ In addition, pure DownBeating Nystagmus (DBN) could be of a central etiology which requires imaging, ${ }^{43}$ while $\mathrm{DBN}$ as a type of positional nystagmus in healthy individuals though uncommon in patients with intact vestibular and central nervous system function, has been reported in several studies. ${ }^{17,44-50}$ Finally, central adaption to BPPV has also been suggested as a cause of nystagmus reversal in BPPV patients. ${ }^{19}$ All these confounding presentations may have resulted in the controversial reported prevalence of AC-BPPV and may suggest that such type of BPPV if seen should be very uncommon. As the anterior canal is at a very steep angle from the sagittal plane $\left(43^{0} \pm 8.7\right),{ }^{51}$ the debris would have to go against gravity in order to remain in the canal, when the patient is in an upright position. It is also difficult to put the canal in the most gravity-dependent position to elicit nystagmus with traditional or modified side-lying Hallpike. On top of excluding atypical nystagmus patterns, no deep head hanging was performed in this study which makes it difficult to assess for anterior canal BPPV (if any). This could also explain why no AC-BPPV was detected in this study. If deep-head hanging was performed in all patients, it is plausible that the study may find close to $3 \%$ of BPPV types as AC$\mathrm{BPPV}$, according to a recent pooled systematic review. ${ }^{20}$

Hypertension was significantly correlated with occurrence of BPPV in the age group of 71-80 years. However, this association may be under or overestimated given the selection bias of case controls, which is not a well representation of the general population. Despite this limitation, hypertension and other comorbidities such as migraine, head injuries, diabetes and cardiovascular disease have been reported to be significantly associated with BPPV in a few studies. ${ }^{52-55}$ This association may be explained by a compromise in the vascular supply to the labyrinth or as a direct concussion to the labyrinth after head injury. As the labyrinth's vascular supply comes from a branch of the basilar artery (Anterior inferior cerebellar artery; AICA), any comorbidities affecting vascular flow to the AICA may result in microvascular insults to the labyrinth and hence, detachment of the otoconia from the utricle. However, it is not known if the association of BPPV with hypertension is due to effects of calcium-channel blockers or the disease itself.

It is thus, not surprising to find a moderate prevalence of migraine (28\%), head injury (24\%), diabetes (21\%) and cardiovascular problems $(19 \%)$ reported in this study. What is less reported however, is the co-existence of anxiety and depression in BPPV patients. ${ }^{56,57}$ The influence of anxiety and depression on BPPV has not been adequately described and in this study, about $20 \%$ of patients with BPPV have co-existing anxiety and depression. Whether these psychiatric comorbidities affect treatment outcomes, recurrence rates and HRQoL in BPPV patients require further study. The association between occurrence of BPPV and results of posturography, SOT composite score and other comorbidities cannot be well estimated due to the under-representation of case-controls. This may be better elucidated with large cohort longitudinal, prospective and randomized case-controlled studies.

Although treatments were carried out by different clinicians, all clinicians had a minimum of a doctoral degree and extensive training on vestibular and equilibrium studies according to the standards of AIB. They were also all exposed to the same training curriculum. As it was hard to track the number of maneuvers performed due to inconsistencies in the reporting, the number of treatment sessions before complete resolution was used instead. Most patients required only 1 treatment session with $97 \%$ of all patients in complete remission within 3 treatment sessions. It is of AIB's protocol to not have more than 3 treatment maneuvers performed in one treatment session. The recurrence rate of almost $17 \%$ though higher than some studies (range; $4-30 \%$ ) are not specific to any one type of treatment used. Hence, some types of treatment maneuvers may have higher recurrence rates, which is not differentiated in this study. Moreover, prior studies reporting recurrence rates only looked at unilateral involvement with single-type treatment. In our study, recurrence rates considered mixed-canal and bilateral canal type BPPV, which have been associated with head trauma and higher recurrence rates.

There is also a high prevalence of falls and the fear of falling in almost half of the patients surveyed ( $48 \% \& 41 \%)$. Of those patients with available postural study results, more than half of them were abnormal (54\%), suggesting an elevated fall risk. Given the high prevalence of falls, fear of falling and abnormal postural stability in patients with BPPV, unrecognized and untreated BPPV may contribute to falls especially in the elderly with multisensory decline. Some studies have also demonstrated the improvement in postural stability after BPPV treatment which may suggest an influence of BPPV on postural stability. ${ }^{58-61}$

\section{Conclusion}

Identifying parameters of BPPV for future investigation, cannot be achieved without laying the groundwork with large cohort demographic analyses such as one outlined in this study. Demographic studies are important as they help to improve recognition and understanding of BPPV. Untreated and unrecognized BPPV not only elevates fall risk but also increases healthcare costs. Hence, despite a good chance of spontaneous resolution, clinicians should treat BPPV as soon as possible to reduce the financial burden on healthcare systems, minimize risk of falls, reduction in HRQoL and disruption to ADLs. BPPV when untreated may also result in self-migration to the horizontal canal, which is more provocative and nauseating to the patients, further limiting their daily activities. Adverse effects of BPPV treatments are uncommon but may include emesis and cervical or spinal discomfort. Hence, choosing the most comfortable and efficacious treatment maneuver is important, to maximize comfort and minimize provocation and nausea with expediency. Having a selection of maneuvers to choose from with comparable efficacy will help in this selection. In this study, comparison of treatment efficacy as a function of treatment type was not established. Hence, future studies should include, large cohort prospective analyses to delineate the efficacy, recurrence rates of different treatment maneuvers, and the relationship between postural stability and BPPV.

\section{Acknowledgements}

The authors would like to thank all staff members of the American Institute of Balance who have made this study possible. 


\section{Conflict of interests}

This research received no specific grant from any funding agency, commercial or not-for-profit sectors. Competing interests: None declared.

\section{Funding}

None.

\section{References}

1. Bath AP, Walsh RM, Ranalli P, et al. Experience from a multidisciplinary “dizzy" clinic. Am J Otol. 2000;21(1):92-97.

2. Gans RE. Overview of BPPV: treatment methodologies. Hear Rev. 2000;7:34-38.

3. Schuknecht HF. Cupulolithiasis. Arch Orolaryngol. 1969;90(6):765778 .

4. Parnes LS, McClure JA. Free-floating endolymph particles: a new operative finding during posterior semicircular canal occlusion. Laryngoscope. 1992;102:988-92.

5. Roberts RA, Gans RE. Background technique (2008), interpretation and usefulness of positional/positioning testing. In G. Jacobsen \& N Shepard (Eds). Balance Function Assessment and Management. San Diego, CA: Plural Publishing; 2008. p. 171-196.

6. Hornibrook J. Benign Paroxysmal Positional Vertigo (BPPV): History, Pathophysiology, Office Treatment and Future Directions. International Journal of Otolaryngology. 2011;2011:1-13.

7. He LL, Li XY, Hou MM, et.al. Association between bone mineral density and benign paroxysmal positional vertigo: a meta-analysis. European Archives of Oto-Rhino-Laryngology. 2019;276;6:1561-1571.

8. Roberts RA, Abrams H, Sembach MK, et.al. Utility Measures of HealthRelated Quality of Life in Patients Treated for Benign Paroxysmal Positional Vertigo. Ear and Hearing. 2009;30; 369-376.

9. Lopez-Escamez JA, Gamiz MJ, Fernandez-Perez A, et.al. Longterm outcome and health-related quality of life in benign paroxysmal positional vertigo. Eur. Arch. Otorhinolaryngol. 2005; 262:507-511.

10. Kao CL, Hsieh WL, Chern CM, et.al. Clinical features of benign paroxysmal positional vertigo (BPPV) in Taiwan: differences between young and senior age groups. Archives of Gerontology and Geriatrics. 2009;49;2:S50-S54.

11. Epley JM. The canalith repositioning procedure: for treatment of benign paroxysmal positional vertigo. Otolaryngol head Neck Surg. 1992;107:399-404

12. Herdman S, Tusa R, Zee D, et.al. Single treatment approaches to benign paroxysmal positional vertigo. Arch Otolarngol Head Neck Surg. 1993;119:450-454.

13. Semont A, Freyss G, Vitte E. Curing the BPPV with a liberatory maneuver. Adv Otolaryngol Rhinol Laryngol. 1988;42:290-293.

14. Roberts RA, Gans RE, Montaudo R. Efficacy of a new treatment maneuver for posterior canal benign paroxysmal positional vertigo. $J$ Am Acd Audiol. 2006;17:598-604.

15. Baloh RW, Honrubia V, Jacobson K. Benign positional vertigo: clinical and oculographic features in 240 cases. Neurology. 1987;37:371-8.

16. Macdonald NK, Kaski D, Saman Y, et.al. Central Positional Nystagmus: A Systematic Literature Review. Front Neurol. 2017;8: 1-10.
17. Roberts RA, Bittel SN, Gans RE. Positional Nystagmus in Patients Evaluated for Dizziness and Imbalance. Advances in Otolaryngology. 2016. p. $1-10$

18. Califano L, Salafia F, Mazzone S, et.al. Anterior canal BPPV and apogeotropic posterior canal BPPV: two rare forms of vertical canalolithiasis. Acta Otorhinolaryngologica Italica. 2014;34(3):189197.

19. Choi SY, Choi KD, Choi Y, et.al. Short-term central adaptation in benign positional paroxysmal vertigo. Journal of the Neurological Sciences. 2017;381(15):748.

20. Anagnostou E, Kouzi I, Spengos K. Diagnosis and Treatment of Anterior-Canal Benign Paroxysmal Positional Vertigo: A Systematic Review. J Clin Neurol. 2015;11(3):262-267.

21. Gans RE, Kurtzer D, McLeod H. New Horizontal Canal Benign Paroxysmal Positional Vertigo Treatment: Kurtzer Hybrid Maneuver. Glob J Otolaryngol. 2017;6(3):1-5.

22. Kao CL, Hsieh WL, Chern CM, et.al. Clinical features of benign paroxysmal positional vertigo (BPPV) in Taiwan: differences between young and senior age groups. Archives of Gerontology and Geriatrics. 2009;49(2):S50-54

23. Moon SY, Kim JS, Kim BK, et.al. Clinical Characteristic of Benign Paroxysmal Positional Vertigo in Korea: A Multicenter Study. J Korean Med Sci. 2006;21:539-43

24. Katsarkas A. Benign Paroxysmal positional vertigo (BPPV): idiopathic versus post-traumatic. Acta Otolaryngo. (Stockh). 1999;119:745-749.

25. Lynn S, Pool A, Rose D, et.al. Randomized trial of the canalith repositioning procedure. Otolaryngol Head Neck Surg. 1995;113: 712720 .

26. Angeli SI, Hawley R, Gomez O. Systematic approach to benign paroxysmal positional vertigo in the elderly. Otolaryngol. Head Neck Surg. 2003;128(5):719-725.

27. Yimtae K, Sirompotong S, Sae-Seaw P. A randomized trial of the canalith repositioning procedure. Laryngoscope. 2003;113(5): 828-832.

28. Steenerson RL, Cronin GW, Marbach PM. Effectiveness of treatment techniques in 923 cases of benign paroxysmal positional vertigo. Laryngoscope. 2005;115(2):226-231.

29. Von Brevern M, Seelig T, Neuhauser H, et.al. Benign paryoxysmal positional vertigo predominantly affects the right labyrinth. $J$ Neurol Neurosurg Psychiatry. 2004;75:1487-8.

30. Gans RE, Harrington-Gans PA. Treatment Efficacy of Benign Paroxysmal Positional Vertigo (BPPV) with Canalith Repositioning Maneuver and Semont Liberatory Maneuver in 376 Patients. Seminars in hearing. 2002;23(2):129-142.

31. Yetiser S, Ince D. Demographic Analysis of Benign Paroxysmal Positional Vertigo as a Common Public Health Problem. Annals of Medical and Health Sciences Research. 2015;5(1):50-53.

32. Balatsouras DG, Koukoutsis G, Fassolis A, et.al. Benign paroxysmal positional vertigo in the elderly: current insights. Clinical Interventions in Ageing. 2018;13:2251-2266.

33. Oghalai JS, Manolidis S, Barth JL, et.al. Unrecognized benign paroxysmal positional vertigo in elderly patients. Otolaryngology-Head and Neck Surgery. 2000;122(5):630-34.

34. University of Florida, Bureau of Economic and Business Research. Florida Population Studies 2015; Bulletin (172); Florida Demographic Estimating Conference, 2015. 
35. Wolf, JS, Boyev KP, Manokey BJ, et.al. Success of the Modified Epley Maneuver in Treating Benign Paroxysmal Positional Vertigo. The Laryngoscope. 1999;109:900-903.

36. Jackson LE, Morgan B, Fletcher-Jr. JC, et.al. Anterior Canal Benign Paroxysmal Positional Vertigo: An Underappreciated Entity. Otology and Neurotology. 2007;28:218-222.

37. Ruckenstein MJ. Therapeutic Efficacy of the Epley Canalith Repositioning Maneuver. The Laryngoscope. 2001;111(6):940-945.

38. Korres SG, Balatsouras DG. Diagnostic, pathophysiologic, and therapeutic aspects of benign paroxysmal positional vertigo. Otolaryngol Head Neck Surg. 2004;131(4):438-44.

39. McClure JA. Horizontal canal benign positional vertigo. J Orolaryngol. 1985;14(1):30-5.

40. Parnes LS, Agrawal SK, Atlas J. Diagnosis and management of benign paroxysmal positional vertigo (BPPV). CMAJ. 2003;169: 681-93.

41. Shim DB, Ko KM, Lee JH, et.al. Natural history of horizontal canal benign paroxysmal positional vertigo is truly short. Journal of Neurology. 2015;262(1):74-80.

42. Vannucchi P, Giannoni B, Pagnini P. Treatment of Horizontal Semicircular Canal Benign Paroxysmal Positional Vertigo. Journal of Vestibular Research. 1997;7(1):1-6.

43. Leigh RJ, Zee DS. The neurology of eye movements. $5^{\text {th }}$ ed. Oxford, UK, Oxford University Press; 2015.

44. H. Barber, G Wright. Positional nystagmus in normal. Advances in OtoRhino-Laryngology. 1973;19:276-285.

45. McAuley JR, Dickman JD, Mustain W, et.al. Positional nystagmus in asymptomatic hunan subjects. Otolaryngology-Head and Neck Surgery. 1996;114(4):543-553.

46. Sunami K, Tochino R, Zushi $\mathrm{T}$, et.al. Positional and positioning nystagmus in healthy subjects under videonystagmoscopy. Acta OtoLaryngologica. Supplementum. 2004;554:35-37.

47. Coats AC. Computer-quantified positional nystagmus in normal American Journal of Otolaryngology-Head and Neck Medicine and Surgery. 1993;14(5):314-326.

48. Johkura K, Momoo T, Juroiwa Y. Positional nystagmus in patients with chronic dizziness. Journal of Neurology, Neurosurgery and Psychiatry. 2008;79(12):1324-1326.

49. Hajioff D, Barr-Hamilton RM, Colledge NR, et.al. Re-evaluation of normative electronystagmography data in healthy ageing. Clinical Otolaryngology and Allied Sciences. 2000;25(4):249-252.
50. Van der Stappen A, Wuyts FL, Van de Heyning PH. Computerized electronystagmography: normative data revisited. Acta OtoLaryngologica. 2000;120(6):724-730.

51. Kim DK, Kim DR, Jeong SH, et.al. Analysis of the coplanarity of functional pairs of semicircular canals using three-dimensional images reconstructed from temporal bone magnetic resonance imaging. The Journal of Laryngology and Otology. 2015;129:430-434.

52. Ogun OA, Janky KL, Cohn ES, et.al. Gender-Based Comorbidity in Benign Paroxysmal Positional Vertigo. PLoS ONE. 2014; 9(9):1-7

53. Stefano AD, Dispenza F, Suarez H, et.al. A multicenter observational study on the role of comorbidities in the recurrent episodes of benign paroxysmal positional vertigo. Auris Nasus Larynx. 2014;41:31-36.

54. Picciotti PM, Lucidi D, De Corso E, et.al. Comorbidities and recurrence of benign paroxysmal positional vertigo: personal experience. International Journal of Audiology. 2016;55(5):279-284.

55. Faralli M, Cipriani L, Del Zompo MR, et.al. Benign paroxysmal positional vertigo and migraine: analysis of 186 cases. B-ENT. 2014;10:133-139.

56. Ferrari S, Monzani D, Baraldi S, et.al. Vertigo ïn the pink": The Impact of Female Gender on Psychiatric-Psychosomatic Comorbidity in Benign Paroxysmal Positional Vertigo Patients. The Academy of Psychosomatic Medicine. 2014;55:280-288.

57. Eckhardt-Henn A, Best C, Bense S, et.al. Psychiatric comorbidity in different organic vertigo syndromes. J Neurol. 2008;255:420-428.

58. Blatt PJ, Georgakakis GA, Herdman SJ, et.al. The Effect of the Canalith Repositioning Maneuver on Resolving Postural Instability in Patients with Benign Paroxysmal Positional Vertigo. Am J Otol. 2000;21(3):35663.

59. Girolamo SD, Paludetti G, Briglia G, et.al. Postural Control in Benign Paroxysmal Positional Vertigo Before and After Recovery. Acta Orolaryngol (Stockh). 1998;118:289-293.

60. Kasse CA, Santana GG, Branco-Barreiro FCA, et.al. Postural Control in Older Patients with Benign Paroxysmal Positional Vertigo. Otolaryngology-Head and Neck Surgery. 2012;146(5): 809-815.

61. Omara A, Mosaad, DM, Mohamed AS, et.al. Epley repositioning maneuver versus Gans repositioning maneuver on postural instability in elderly patients with benign paroxysmal positional vertigo. The Egyptian Journal of Otolarngology. 2017;33:518-522. 